\title{
EXTREMAL DEGREE-PRODUCT INDICES OF GRAPHS WITH FIXED NUMBER OF PENDANT VERTICES AND CYCLOMATIC NUMBER
}

\author{
M. K. JAMIL*, I. TOMESCU, \\ N. AKHTER MUHAMMAD KAMRAN JAMIL \\ Abdus Salam School of Mathematical Sciences GCU, Lahore, Pakistan. \\ 68-B New Muslim Town, Lahore, Pakistan.
}

Email: akhtarnaweed@yahoo.com,m.kamran.sms@gmail.com

\begin{abstract}
The Narumi-Katayama index $N K(G)$ and first multiplicative Zagreb index $\prod_{1}(G)$ of a graph $G$ are defined as the product of the degrees of the vertices of $G$ and the product of square of the degrees of the vertices of $G$, respectively. The second multiplicative Zagreb index is defined as $\prod_{2}(G)=\prod_{u v \in E(G)} d(u) d(v)$. In this paper, we compute the extremal $N K(G), \prod_{1}(G)$
\end{abstract} and $\prod_{2}(G)$ for the graphs with given order, number of pendant vertices and cyclomatic number.

\section{INTRODUCTION}

In this paper we are concerned with simple connected graphs. Let $G$ be such a graph with vertex set $V(G)$ and edge set $E(G)$. The number of vertices and edges of $G$ are denoted by $n$ and $m$, respectively. In a graph $G$ the number of independent cycles is called its cyclomatic number, denoted by $\gamma$. For connected graphs, the cyclomatic number is equal to $\gamma=m-n+1$. Recall that graphs with $\gamma=0,1,2$ are referred to as trees, unicyclic graphs, and bicyclic graphs, respectively. Let $v \in V(G)$ then the degree of $v$, denoted as $d(v)$, is the number of vertices of $G$ adjacent to $v$. A vertex $v$ with $d(v)=1$ is called a pendant vertex. A graph with $n$ vertices and $n_{1}$ pendant vertices will be said to be an $\left(n, n_{1}\right)$-graph [2].

In 1984, Narumi and Katayama [6] established a definition "simple topological index":

$$
N K(G)=\prod_{v \in V(G)} d(v)
$$

In recent works on this graph invariant $[1,4,10]$, the name Narumi-Katayama index is being used. In [13] You and Liu deduced extremal $N K(G)$ of trees, unicyclic graphs with given diameter and vertices and the minimal $N K(G)$ of bicyclic graphs with given vertices was obtained.

The vertex-degree-based graph invariants

$$
\begin{aligned}
& M_{1}(G)=\sum_{v \in V(G)} d(v)^{2} \\
& M_{2}(G)=\sum_{u v \in E(G)} d(u) d(v)
\end{aligned}
$$

are known under the name first and second Zagreb index, respectively. They have been conceived in the 1970s and found considerable applications in chemistry [7, 11]. The Zagreb indices were subject to a large number of mathematical studies, of which we mention only a few newest [3, 5, 14]. Todeschini et al. [8,9] have recently proposed to consider the multiplicative variants of additive graph invariants, which applied to the Zagreb indices would lead to 


$$
\begin{aligned}
& \prod_{1}(G)=\prod_{v \in V(G)} d(v)^{2} \\
& \prod_{2}(G)=\prod_{u v \in E(G)} d(u) d(v)=\prod_{v \in V(G)} d(v)^{d(v)}
\end{aligned}
$$

The properties of these "multiplicative Zagreb indices" have not been studied so far, and the present work is an attempt to contribute towards their better understanding.

In [2], I. Gutman et al. computed the minimal first Zagreb index of graphs with fixed number of pendant vertices. In this paper we determine the extremal values of the Narumi-Katayama, first Zagreb and second Zagreb indices of connected $\left(n, n_{1}\right)$-graphs with fixed cyclomatic number and show that these bounds are tight. For other notation in this paper we refer [12].

\section{EXTREMAL $\left(n, n_{1}\right)$-GRAPHS RELATIVELY TO NARUMI-KATAYAMA INDEX}

We need the following well-known property.

Lemma 2.1 Let $n, r, x_{1}, x_{2}, \ldots, x_{n}$ be positive integers such that $x_{i} \geq r$ and

$$
\sum_{i=1}^{n} x_{i}=a \geq r n
$$

Then $\prod_{i=1}^{n} x_{i}$ is minimum if and only if there exists an index $i, 1 \leq i \leq n$ such that $x_{i}=a-(n-1) r$ and $x_{j}=r$ for every $j \neq i$ and it is maximum if and only if $x_{1}, \ldots, x_{n}$ are almost equal, i.e., $\max \left\{x_{1}, \ldots, x_{n}\right\}-\min \left\{x_{1}, \ldots, x_{n}\right\} \leq 1$.

Since in a connected graph a non-pendant vertex has at least degree 2 and $N K(G)=\prod_{x \in V(G), d(x) \geq 2} d(x)$, by Lemma 2.1 we have the following consequence:

Corollary 2.2 If $G$ is a connected graph of order $n$ and size $m$ with $n_{1}$ pendant vertices $\left(n>n_{1}\right)$ then

$$
N K(G) \geq 2^{n-n_{1}-1}\left(2 m-2 n+n_{1}+2\right)
$$

This bound is achieved if $G$ has one vertex of degree $2 m-2 n+n_{1}+2$ and all other non-pendant vertices are of degree 2 .

Let $G$ be a connected $\left(n, n_{1}\right)$-graph with cyclomatic number $\gamma$. If $\gamma=0$ then $G$ is a tree and $2 \leq n_{1} \leq n-1$. Otherwise we suppose that $0 \leq n_{1} \leq n-1$. We define the auxiliary quantities $t$, $n_{t}$ and $n_{t+1}$ as:

$$
\begin{aligned}
& t=\left\lfloor\frac{n+2(\gamma-1)}{n-n_{1}}\right\rfloor+1 \quad, \quad n_{t}=\left(n-n_{1}\right)\left\lfloor\frac{n+2(\gamma-1)}{n-n_{1}}\right\rfloor-n_{1}-2(\gamma-1) \\
& n_{t+1}=n-\left(n-n_{1}\right)\left\lfloor\frac{n+2(\gamma-1)}{n-n_{1}}\right\rfloor+2(\gamma-1) .
\end{aligned}
$$

Recall that $\lfloor x\rfloor$ is the greatest integer that is not greater then $x$, or the integer part of $x$. 
Theorem 2.3 Let $G$ be a connected $\left(n, n_{1}\right)$-graph with cyclomatic number $\gamma$. Then

$$
2^{n-n_{1}-1}\left(2 \gamma+n_{1}\right) \leq N K(G) \leq\left(\left\lfloor\frac{n+2(\gamma-1)}{n-n_{1}}\right\rfloor+1\right)^{n_{t}}\left(\left\lfloor\frac{n+2(\gamma-1)}{n-n_{1}}\right\rfloor+2\right)^{n_{t+1}} .
$$

(a) For trees $(\gamma=0)$ both lower and upper bounds are reached.

(b) For $\gamma \geq 1$ lower bound can be attained for $n \geq 2 \gamma+1+n_{1}$ and upper bound for $n \geq 3 \gamma+n_{1}$.

Proof. Lower bound. A graph with $n$ vertices and cyclomatic number $\gamma$ has size $m=n+\gamma-1$, so by Corollary 2.2 , we have

$$
N K(G) \geq 2^{n-n_{1}-1}\left(2 \gamma+n_{1}\right) .
$$

To see that this bound can be reached for $\gamma=0$ consider a path with $n-n_{1}+1$ vertices and add $n_{1}-1$ pendant vertices, all adjacent to a unique end vertex of this path.

For $\gamma>0$ take $\gamma$ cycles, having together $n-n_{1}$ vertices and a unique common vertex. Then all $n_{1}$ remaining vertices are joined each by an edge to this common vertex. It follows that $n \geq 2 \gamma+1+n_{1}$, and equality holds when all $\gamma$ cycles have a length equal to 3 . Figures 1,3 and 5 illustrate graphs having minimum $N K$ index for $n=22, n_{1}=13$ and $\gamma=0,1,2$.

Upper bound. By Lemma $2.1 N K(G)$ will be maximum if $G$ has $n_{t} \quad\left(0<n_{t} \leq n-n_{1}\right)$ non-pendant vertices of degree $t$ and $n_{t+1}=n-n_{1}-n_{t}$ non-pendant vertices of degree $t+1$, then

$$
N K(G) \leq t^{n} t(t+1)^{n} t+1 \text {. }
$$

As a graph of order $n$ with cyclomatic number $k$ has size $n+\gamma-1$, we can write:

which yields

$$
n_{1}+t n_{t}+(t+1)\left(n-n_{1}-n_{t}\right)=2(n+\gamma-1),
$$

$$
t\left(n-n_{1}\right)-n_{t}=n+2(\gamma-1),
$$

or

$$
t-\frac{n_{t}}{n-n_{1}}=\frac{n+2(\gamma-1)}{n-n_{1}} .
$$

Taking integer parts,

$$
\left\lfloor t-\frac{n_{t}}{n-n_{1}}\right\rfloor=\left\lfloor\frac{n+2(\gamma-1)}{n-n_{1}}\right\rfloor .
$$

Since $t$ is a positive integer, we obtain

$$
t-1=\left\lfloor\frac{n+2(\gamma-1)}{n-n_{1}}\right\rfloor,
$$

or

$$
t=\left\lfloor\frac{n+2(\gamma-1)}{n-n_{1}}\right\rfloor+1
$$

From equations (3) and (4),

which gives

$$
\left(n-n_{1}\right)\left\lfloor\frac{n+2(\gamma-1)}{n-n_{1}}\right\rfloor+n-n_{1}-n_{t}=n+2(\gamma-1)
$$




$$
n_{t}=\left(n-n_{1}\right)\left\lfloor\frac{n+2(\gamma-1)}{n-n_{1}}\right\rfloor-n_{1}-2(\gamma-1) .
$$

As $n_{t+1}=n-n_{1}-n_{t}$, we get:

$$
n_{t+1}=n+2(\gamma-1)-\left(n-n_{1}\right)\left\lfloor\frac{n+2(\gamma-1)}{n-n_{1}}\right\rfloor .
$$

From equations (1) and (4) we deduce:

$$
N K(G) \leq\left(\left\lfloor\frac{n+2(\gamma-1)}{n-n_{1}}\right\rfloor+1\right)^{n} t\left(\left\lfloor\frac{n+2(\gamma-1)}{n-n_{1}}\right\rfloor+2\right)^{n_{t+1}}
$$

as required.

For $\gamma=0$ the upper bound can be reached. To see this consider a path $P_{n-n_{1}}$ with $n-n_{1}$ vertices. Now add the remaining $n_{1}$ pendant vertices using the following algorithm: join each new vertex sequentially, to a vertex of $P_{n-n_{1}}$, having minimum degree. Initially, all vertices have degrees 1 and 2 and after that we obtain, by construction, a tree with $n_{1}$ pendant vertices and non-pendant vertices having almost equal degrees.

For $\gamma>0$ take $\gamma$ vertex disjoint cycles containing together $n-n_{1}$ vertices and joined by edges, such that by contracting each cycle to a vertex yields a path with $\gamma$ vertices. Then join each new vertex sequentially, to a vertex on the cycles, having minimum degree. Initially all degrees are 2 and 3 and after that we obtain, by construction, almost equal degrees for non-pendant vertices. We have $n \geq 3 \gamma+n_{1}$, and equality holds when all vertex disjoint cycles have a length equal to 3 . Figures 2, 4 and 6 illustrate graphs having maximum $N K$ index for $n=22, n_{1}=13$ and $\gamma=0,1,2$.

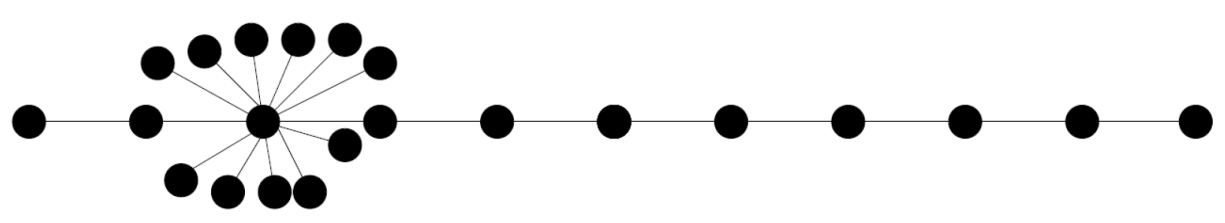

Figure 1: Tree with $n=22$ and $n_{1}=13$ having minimal NK index.

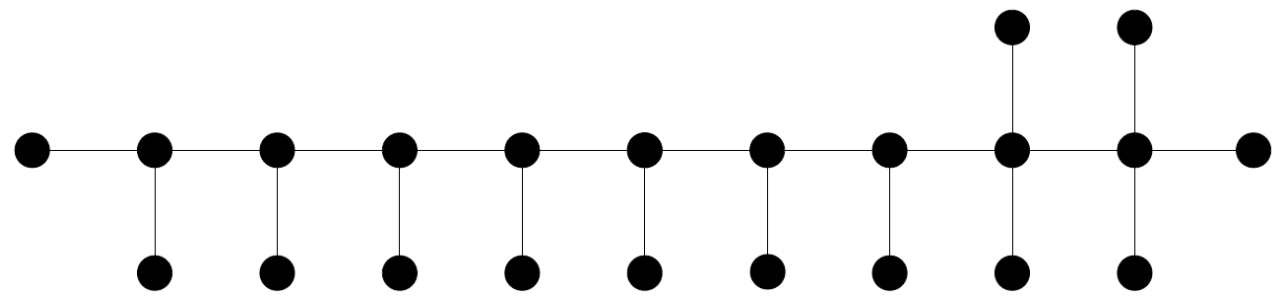

Figure 2: Tree with $n=22$ and $n_{1}=13$ having maximal NK index. 


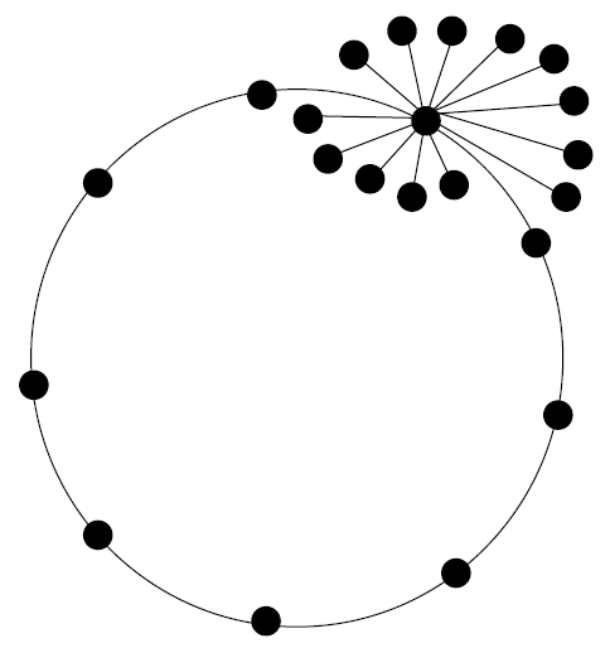

Figure 3: Unicyclic graph with $n=22$ and $n_{1}=13$ having minimal NK index.

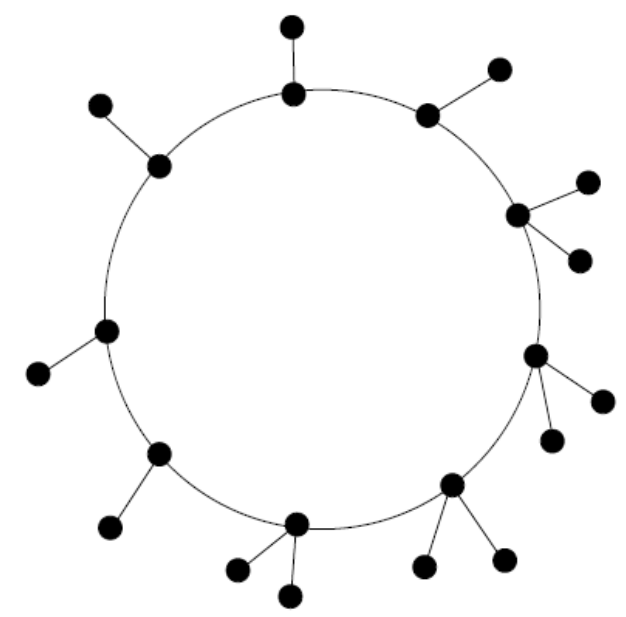

Figure 4: Unicyclic graph with $n=22$ and $n_{1}=13$ having maximal NK index.

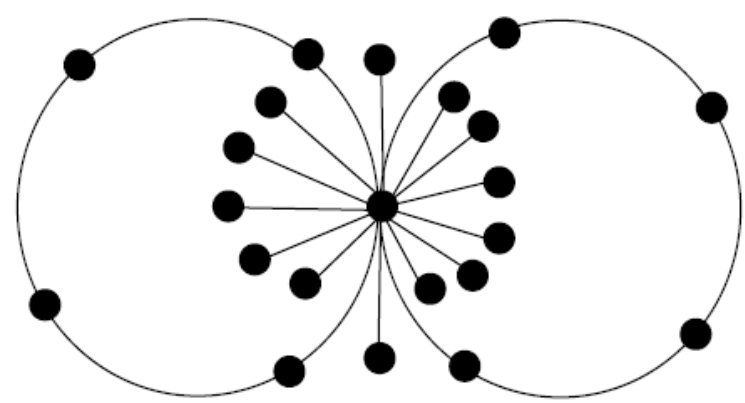

Figure 5: Bicyclic graph with $n=22$ and $n_{1}=13$ having minimal NK index. 


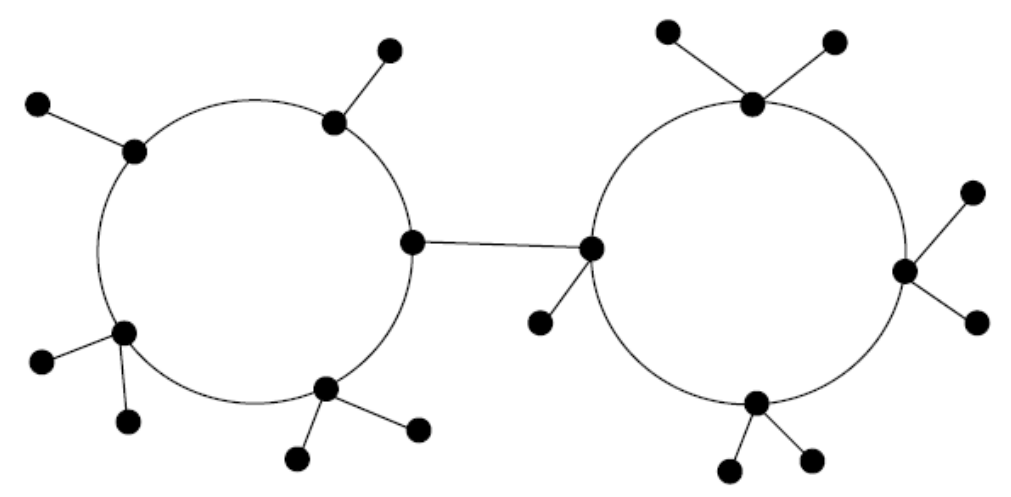

Figure 6: Bicyclic graph with $n=22$ and $n_{1}=13$ having maximal NK index.

\section{Extremal $\left(n, n_{1}\right)$-graphs relatively to Multiplicative Zagreb indices}

Since $\prod_{1}(G)=N K(G)^{2}$, Theorem 2.3 implies the following corollary.

Corollary 3.1 Let $G$ be a connected $\left(n, n_{1}\right)$-graph with cyclomatic number $\gamma$. Then

$$
4^{n-n_{1}-1}\left(2 \gamma+n_{1}\right)^{2} \leq \prod_{1}(G) \leq\left(\left\lfloor\frac{n+2(\gamma-1)}{n-n_{1}}\right\rfloor+1\right)^{2 n_{t}}\left(\left\lfloor\frac{n+2(\gamma-1)}{n-n_{1}}\right\rfloor+2\right)^{2 n_{t+1}} .
$$

(a) For trees $(\gamma=0)$ both lower and upper bounds are reached.

(b) For $\gamma \geq 1$ lower bound can be attained for $n \geq 2 \gamma+1+n_{1}$ and upper bound for $n \geq 3 \gamma+n_{1}$.

Some extremal properties of the second multiplicative Zagreb index in some families of graphs are deduced below. First we need the following property:

Lemma 3.2 Function $\phi(x)=\frac{x^{x}}{(x-1)^{x-1}}$ is increasing for $x \geq 2$.

Proof. We get

$$
\phi^{\prime}(x)=\frac{x^{x}(x-1)^{x-1}(\ln x-\ln (x-1))}{(x-1)^{2(x-1)}}>0
$$

therefore $\phi$ is increasing for $x \geq 2$.

Let $\Gamma_{n, n_{1}}$ be the family of connected graphs with order $n$ and $n_{1}$ pendant vertices.

We define a family of trees of order $n$ with $n_{1}$ pendant vertices, denoted $\mathrm{T}_{n, n_{1}}^{*}$ as the set of trees of order $n$ consisting of $n_{1}$ paths having a common end vertex. Note that $\mathrm{T}_{n, 2}^{*}=\left\{P_{2}\right\}$.

Theorem 3.3 Let $T$ be a tree in $\Gamma_{n, n_{1}}$, where $n \geq n_{1} \geq 2$; then

$$
\prod_{2}(T) \leq n_{1}^{n_{1}} 4^{n-n_{1}-1}
$$

and the equality holds if and only if $T \in \mathrm{T}_{n, n_{1}}^{*}$. 
Proof. We shall prove this result by induction on $n+n_{1}$. Let $f\left(n, n_{1}\right)=n_{1}^{n_{1} 4^{n-n_{1}-1}}$. If $n_{1}=2$ and $n \geq n_{1}$, then $T \cong P_{n}$ and by direct calculation $\prod_{2}\left(P_{n}\right)=4^{n-2}$ and this equals $f(n, 2)$, hence the property is verified.

Let $n_{1} \geq 3$ and suppose that the result is true for any tree of order $n^{\prime}$ with $n_{1}^{\prime}$ pendant vertices such that $7 \leq n^{\prime}+n_{1^{\prime}}<n+n_{1}$. Let $T$ be a tree in $\Gamma_{n, n_{1}}$ and $x$ be a pendant vertex of $T$. If $x y \in E(T)$, suppose that $d(y)=a$. We shall consider two cases: 1) $a=2$ and 2) $a \geq 3$.

1) In this case $T-x$ has order $n-1$ and $n_{1}$ pendant vertices, hence $\prod_{2}(T)=2^{2} \prod_{2}(T-x)$ and by the induction hypothesis $\prod_{2}(T-x) \leq f\left(n-1, n_{1}\right)$ and the equality holds if and only if $T-x \in \mathrm{T}_{n-1, n_{1}}^{*}$. It follows that $\prod_{2}(T) \leq f\left(n, n_{1}\right)$ and the equality holds if and only if $T \in \mathrm{T}_{n, n_{1}}^{*}$.

2) $T-x$ having order $n-1$ and $n_{1}-1$ pendant vertices, we have

$$
\prod_{2}(T)=\frac{a^{a}}{(a-1)^{a-1}} \prod_{2}(T-x) .
$$

By our supposition of induction,

$$
\prod_{2}(T) \leq \frac{a^{a}}{(a-1)^{a-1}} f\left(n-1, n_{1}-1\right)
$$

Equality holds if and only if $T-x \in \mathrm{T}_{n-1, n_{1}-1}^{*}$. The last inequality may be written

$$
\prod_{2}(T) \leq \frac{a^{a}}{(a-1)^{a-1}} \frac{\left(n_{1}-1\right)^{n_{1}-1}}{n_{1}^{n_{1}}} f\left(n, n_{1}\right)
$$

By Lemma 3.1, $\phi(x)$ is an increasing function, so $\frac{a^{a}}{(a-1)^{a-1}}$ is maximum for maximum value of $a$ and in the set of trees with $n_{1}$ pendant vertices the maximum degree of a vertex is $n_{1}$, so

$$
\prod_{2}(T) \leq f\left(n, n_{1}\right) \text {. }
$$

Equality holds if and only if $T \in \mathrm{T}_{n, n_{1}}^{*}$ since $d(y)=n_{1}$ only if $x$ is adjacent to the unique vertex in $T-x$ of degree $n_{1}-1$.

We define a family of unicyclic graphs of order $n$ with $n_{1}$ pendant vertices, denoted $U_{n, n_{1}}^{*}$ , as the set of unicyclic graphs of order $n$ consisting of a cycle $C_{p}(p \geq 3)$ and $n_{1}$ paths having a common end vertex which lies on $C_{p}$.

Theorem 3.4 Let $U$ be a unicyclic graph in $\Gamma_{n, n_{1}}$ such that $n>n_{1} \geq 0$; then

$$
\prod_{2}(U) \leq\left(n_{1}+2\right)^{n_{1}+2} 4^{n-n_{1}-1}
$$

and the equality holds if and only if $U \in U_{n, n_{1}}^{*}$.

Proof. We shall prove this result also by induction on $n+n_{1}$. Let $g\left(n, n_{1}\right)=\left(n_{1}+2\right)^{n_{1}+2} 4^{n-n_{1}-1}$. If $n_{1}=0$, then $U \cong C_{n}$ and by direct calculation $\prod_{2}\left(C_{n}\right)=4^{n}=g(n, 0)$. 
Let $n_{1} \geq 1$ and suppose that the result is true for any unicyclic graph of order $n^{\prime}$ with $n_{1^{\prime}}$ pendant vertices, such that $4 \leq n^{\prime}+n_{1^{\prime}}<n+n_{1}$. As before, let $U$ be a unicyclic graph in $\Gamma_{n, n_{1}}$ and $x$ a pendant vertex of $U$. If $y$ is adjacent to $x$ in $U$, let $d(y)=a$. We shall consider two cases: 1) $a=2$ and 2) $a \geq 3$.

1) In this case $U-x$ is unicyclic, has order $n-1$ and $n_{1}$ pendant vertices, hence $\prod_{2}(U)=2^{2} \prod_{2}(U-x)$ and by the induction hypothesis $\prod_{2}(U-x) \leq g\left(n-1, n_{1}\right)$ and the equality holds if and only if $U-x \in U_{n-1, n_{1}}^{*}$. It follows that $\prod_{2}(U) \leq g\left(n, n_{1}\right)$ and the equality holds if and only if $U \in U_{n, n_{1}}^{*}$.

2) $U-x$ having order $n-1$ and $n_{1}-1$ pendant vertices, we get

$$
\prod_{2}(U)=\frac{a^{a}}{(a-1)^{a-1}} \prod_{2}(U-x) .
$$

By induction hypothesis,

$$
\prod_{2}(U) \leq \frac{a^{a}}{(a-1)^{a-1}} g\left(n-1, n_{1}-1\right),
$$

and equality holds if and only if $U \in U_{n-1, n_{1}-1}^{*}$ We deduce

$$
\prod_{2}(U) \leq \frac{a^{a}}{(a-1)^{a-1}} \frac{\left(n_{1}+1\right)^{n_{1}+1}}{\left(n_{1}+2\right)^{n_{1}+2}} g\left(n, n_{1}\right) .
$$

By Lemma 3.1, $\phi(x)$ is strictly increasing in $a$ and in the set of unicyclic graphs with $n_{1}$ pendant vertices the maximum degree of a vertex is $n_{1}+2$, so

$$
\prod_{2}(U) \leq g\left(n, n_{1}\right) \text {. }
$$

Equality holds if and only if $U \in U_{n, n_{1}}^{*}$ since $d(y)=n_{1}+2$ only if $x$ is adjacent to the unique vertex in $U-x$ of degree $n_{1}+1$, which is common to the cycle and $n_{1}-1$ pendant paths.

In a similar way we can generalize this result for a given cyclomatic number $\gamma \geq 2$ for every $n \geq 3 \gamma+n_{1}$ as follows: Let $G_{n, n_{1}, \gamma}$ be the set of connected graphs of order $n$, having $n_{1}$ pendant vertices and cyclomatic number $\gamma$, consisting of $\gamma$ cycles having a common vertex $w$ and $n_{1}$ paths having an end vertex in $w$. If $G$ is a connected graph of order $n$, having $n_{1}$ pendant vertices and cyclomatic number $\gamma$, then

$$
\prod_{2}(G) \leq\left(n_{1}+2 \gamma\right)^{n_{1}+2 \gamma} 4^{n-n_{1}-1}
$$

and the equality holds if and only if $G \in G^{*}{ }_{n, n_{1}, \gamma}$.

\section{Muhammad Kamran Jamil}

Abdus Salam School of Mathematical Sciences, Government College University, Lahore, Pakistan

Email:m.kamran.sms@gmail.com 


\section{Ioan Tomescu}

Faculty of Mathematics and Computer Science, University of Bucharest,

Str. Academiei, 14, 010014 Bucharest, Romania.

Email: ioan@fmi.unibuc.ro

\section{Naveed Akhter}

Abdus Salam School of Mathematical Sciences,

Government College University, Lahore, Pakistan

Email: akhtarnaweed@yahoo.com

\section{References}

[1] I. Gutman, M. Ghorbani, Some properties of the Narumi-Katayama index, Applied Mathematics Letters, 25(2012) 1435-1438.

[2] I. Gutman, M. K. Jamil, N. Akhter, Graphs with fixed number of pendant vertices and minimal first Zagreb index, Trans Comb., 4(2015) 29-34.

[3] B. Horoldagva, S.G. Lee, Comparing Zagreb indices for connected graphs, Discr. Appl. Math., 158 (2010) 1073-1078.

[4] D. J. Klein, V. R. Rosenfeld, The degree-product index of Narumi and Katayama. MATCH Commun. Math. Comput. Chem., 64(2010) 607-618.

[5] M. Khalifeh, H. Yousefi Azari, A.R. Ashrafi, The first and second Zagreb indices of graph operations. Discr. Appl. Math., 157 (2009) 804-811.

[6] H. Narumi, M. Katayama, Simple topological index, A newly devised index characterizing the topological nature of structural isomers of saturated hydrocarbons, Memoirs of the Faculty of Engineering, Hokkaido University 16 (1984) 209-214.

[7] S. Nikoli c', G. Kova č evi c' , A. Mili č evi c' , N. Trinajsti c' , The Zagreb indices 30 years after, Croat. Chem. Acta, 76 (2003) 113-124.

[8] R. Todeschini, D. Ballabio, V. Consonni, Novel molecular descriptors based on functions of new vertex degrees. In: Novel molecular structure descriptors - Theory and applications I. (I. Gutman, B. Furtula, eds.), pp. 73-100. Kragujevac: Univ. Kragujevac 2010.

[9] R. Todeschini, V. Consonni, New local vertex invariants and molecular descriptors based on functions of the vertex degrees. MATCH Commun. Math. Comput. Chem., 64 (2010) 359-372.

[10] Ž. Tomovi c', I. Gutman, Narumi-Katayama index of phenylenes, J. Serb. Chem. Soc., 66 (2001) 243-247.

[11] N. Trinajsti c' , S. Nikoli c' , A. Mili č evi c' , I. Gutman, On Zagreb indices. Kemija u industriji, 59 (2010) 577-589 (in Croatian).

[12] D. B. West, Introduction to Graph Theory, Prentice Hall, Upper Saddle River, 2001.

[13] Z. You, B. Liu, On the extremal Narumi-Katayama index of graphs, Filomat, 28(2014) 523-529.

[14] Q. Zhao, S. Li, On the maximum Zagreb index of graphs with $k$ cut vertices. Acta Appl. Math., 111 (2010) 93-106. 